\title{
Crystal Structure of 11,12,23,24-Tetradehydro-5,6,9,10,17,18,21,22-octahydro- 7H,19H-dibenzo[e,o][1,11]dioxacycloeicosin-7,19-dione
}

\author{
Pablo Wessig, ${ }^{\dagger}$ Charlotte Pick, and Burkhard ZiEMer \\ Institut für Chemie, Humboldt-Universität zu Berlin, Brook-Taylor-Str. 2, D-12489 Berlin
}

\begin{abstract}
The title compound, bearing a twenty-membered ring, was synthesized by macrolactonization of a hydroxy carboxylic acid. The structure was investigated by X-ray crystallography. The compound crystallizes in the monoclinic system, space group $P 2_{1} / c$. In the crystal, two remarkable kinds of interactions between two ester groups and between two arylacetylene moieties were detected.
\end{abstract}

(Received July 7, 2006; Accepted September 25, 2006; Published on web November 16, 2006)

The preparation of macrocyclic compounds is still a challenge for synthetic organic chemistry. Because the formation of large rings consisting of 10 or more atoms is entropically very unfavorable, most of the successfully performed macrocyclizations rely either on large dilution (the Ruggli-Ziegler dilution principle) or on template effects. On the other hand, spontaneous ring closure to conformationally flexible macrocyclic compounds under thermodynamically controlled conditions has been rarely observed. The state of the art concerning macrolactonization in the synthesis of natural products has been collected very recently in an excellent review. ${ }^{1}$

In connection with one of our current research projects, an investigation of the Photo-Dehydro-Diels-Alder (PDDA) reaction, ${ }^{2,3}$ we were interested in the preparation of tenmembered macrocyclic lactones $\mathbf{2}$ from hydroxycarboxylic acid 1. Surprisingly, under acidic conditions (cat. $\mathrm{TsOH} \cdot \mathrm{H}_{2} \mathrm{O}$ in toluene) not the desired lactone $\mathbf{2}$, but the dilactone $\mathbf{3}$, bearing a twenty-membered ring, was formed in moderate yield. We suppose that the two planar and rigid aryl acetylene moieties are responsible for the unexpected result. Obviously, the twentymembered ring in $\mathbf{3}$ is more favorable than the ten-membered ring in $\mathbf{2}$ as well as polymerization products. Here, the crystal structure of $\mathbf{3}$ was analyzed. The formation of $\mathbf{2}$ could be forced

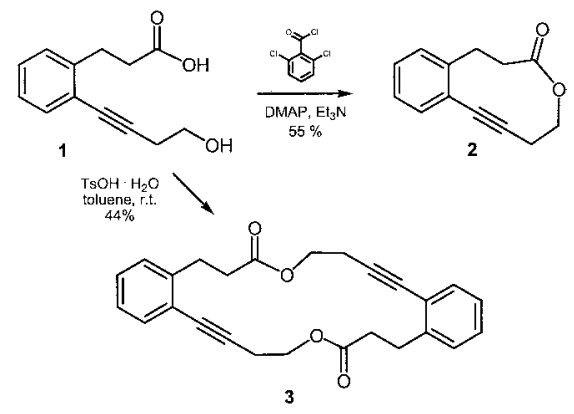

Fig. 1 Formation of lactone $\mathbf{2}$ and dilactone $\mathbf{3}$ from hydroxy carboxylic acid $\mathbf{1}$.

$\doteqdot$ To whom correspondence should be addressed. Email: pablo.wessig@chemie.hu-berlin.de with a macrocyclization method previously described by Yamaguchi et al. ${ }^{4}$

The title compound $\mathbf{3}$ adopts an angle-like geometry in crystals, as can be seen in Fig. 2. The planar regions at both

Table 1 Crystal and experimental data of $\mathbf{3}$

\begin{tabular}{ll}
\hline Empirical formula & $\mathrm{C}_{26} \mathrm{H}_{24} \mathrm{O}_{4}$ \\
Formula weight & 400.45 \\
Temperature & $180(2) \mathrm{K}$ \\
Wavelength & $0.71073 \AA$ \\
Crystal system, space group & monoclinic, $P 2_{1} / c$ \\
Unit cell dimensions & $a=9.9553(15) \AA$ \\
& $b=9.7622(9) \AA \quad \beta=98.256(18)^{\circ}$ \\
& $c=23.145(3) \AA$ \\
Volume & $2226.1(5) \AA^{3}$ \\
$Z$, Calculated density & $4,1.195 \mathrm{Mg}^{3} \mathrm{~m}^{3}$ \\
Absorption coefficient & $0.080 \mathrm{~mm}^{-1}$ \\
$F(00$ 0) & 848 \\
Crystal size & $0.48 \times 0.44 \times 0.28 \mathrm{~mm}^{3}$ \\
Theta range for data collection & 2.27 to $25.22^{\circ}$ \\
Limiting indices & $-11 \leqslant h \leqslant 11,-11 \leqslant k \leqslant 11,-27 \leqslant l$ \\
& $\leqslant 27$ \\
Reflections collected/unique & $14815 / 4032[R($ int $)=0.1172]$ \\
Completeness to theta $=25.22$ & $99.6 \%$ \\
Max. and min. transmission & 0.9780 and 0.9627 \\
Absorption correction & none \\
Data/restraints/parameters & $4032 / 0 / 345$ \\
Goodness-of-fit on $F^{2}$ & 1.068 \\
Final $R$ indices $[I>2 \sigma(I)]$ & $R 1=0.0856, w R 2=0.2359$ \\
$R$ indices (all data) & $R 1=0.1100, w R 2=0.2538$ \\
Extinction coefficient & $0.011(3)$ \\
Largest difmap peak and hole & 0.596 and -0.337 e. $\AA^{-3}$ \\
Measurement & Stoe IPDS \\
Program system & Stoe X-AREA \\
Structure solution & direct methods, SHELXS-97 \\
Refinement method & full-matrix on $F^{2}, \mathrm{SHELXL-97}$ \\
&
\end{tabular}

Standard deviations are given as the last significant figures in parentheses throughout the paper. All hydrogen atoms could be located in difmaps and refined by the least-squares method. The rather high values of R(int) and R1 are caused by crystal twinning. The complete data for this X-ray analysis have been deposited at Cambridge Crystallographic Data Centre under CCDC 620203. 


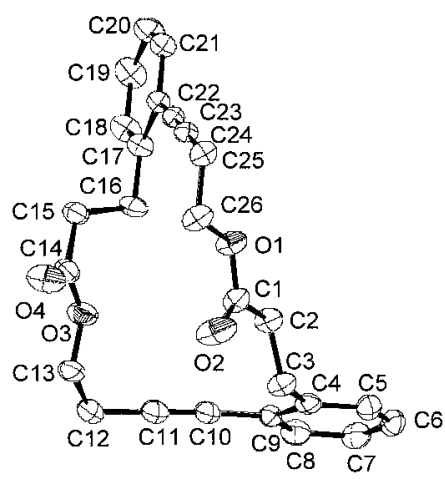

Fig. 2 Molecular structure of compound 3. The probability level of thermal ellipsoids is 50\%. Hydrogen atoms are omitted for clarity.

Table 2 Atomic coordinates $\left(\times 10^{4}\right)$ and equivalent isotropic displacement parameters $\left(\AA^{2} \times 10^{3}\right)$. $U_{\text {(eq) }}$ is defined as one third of the trace of the orthogonalized $U_{i j}$ tensor

\begin{tabular}{|c|c|c|c|c|}
\hline & $x$ & $y$ & $z$ & $U_{(\mathrm{eq})}$ \\
\hline $\mathrm{Cl}$ & $3388(4)$ & $2275(5)$ & $2039(2)$ & $33(1)$ \\
\hline $\mathrm{C} 2$ & $1877(4)$ & $2485(5)$ & 1984(2) & $35(1)$ \\
\hline $\mathrm{C} 3$ & $1445(4)$ & $3621(5)$ & $2368(2)$ & $34(1)$ \\
\hline $\mathrm{C} 4$ & $-57(4)$ & $3899(4)$ & $2247(2)$ & $30(1)$ \\
\hline C5 & $-945(4)$ & $3273(5)$ & $2574(2)$ & $35(1)$ \\
\hline $\mathrm{C} 6$ & $-2324(4)$ & $3483(5)$ & $2454(2)$ & $37(1)$ \\
\hline C7 & $-2863(4)$ & $4334(5)$ & $2000(2)$ & $40(1)$ \\
\hline $\mathrm{C} 8$ & $-2010(4)$ & $4961(5)$ & $1664(2)$ & $38(1)$ \\
\hline $\mathrm{C} 9$ & $-599(4)$ & $4748(4)$ & $1781(2)$ & $29(1)$ \\
\hline $\mathrm{C} 10$ & $277(4)$ & $5409(4)$ & $1424(2)$ & $36(1)$ \\
\hline $\mathrm{C} 11$ & $1017(5)$ & $5999(5)$ & $1148(2)$ & $42(1)$ \\
\hline $\mathrm{C} 12$ & $1953(6)$ & $6725(5)$ & $823(3)$ & $52(1)$ \\
\hline $\mathrm{C} 13$ & $3287(5)$ & $5977(5)$ & $830(3)$ & $48(1)$ \\
\hline $\mathrm{C} 14$ & $3990(4)$ & $3977(4)$ & $399(2)$ & $36(1)$ \\
\hline $\mathrm{C} 15$ & $3578(4)$ & $2807(4)$ & $-4(2)$ & $33(1)$ \\
\hline $\mathrm{C} 16$ & $2227(4)$ & $2106(4)$ & $74(2)$ & $34(1)$ \\
\hline $\mathrm{C} 17$ & $1994(4)$ & $867(4)$ & $-308(2)$ & $30(1)$ \\
\hline $\mathrm{C} 18$ & $1113(4)$ & $905(5)$ & $-839(2)$ & $40(1)$ \\
\hline C19 & $917(5)$ & $-216(5)$ & $-1203(2)$ & $42(1)$ \\
\hline $\mathrm{C} 20$ & $1622(5)$ & $-1423(5)$ & $-1055(2)$ & $39(1)$ \\
\hline $\mathrm{C} 21$ & $2527(4)$ & $-1486(4)$ & $-534(2)$ & $32(1)$ \\
\hline $\mathrm{C} 22$ & $2709(4)$ & $-366(4)$ & $-167(2)$ & $26(1)$ \\
\hline $\mathrm{C} 23$ & $3627(4)$ & $-433(4)$ & $375(2)$ & $25(1)$ \\
\hline $\mathrm{C} 24$ & $4367(4)$ & $-440(4)$ & $833(2)$ & $27(1)$ \\
\hline C25 & $5208(4)$ & $-416(5)$ & $1408(2)$ & $32(1)$ \\
\hline $\mathrm{C} 26$ & $5118(4)$ & $929(5)$ & $1719(2)$ & $37(1)$ \\
\hline 01 & $3698(3)$ & $1187(3)$ & $1741(1)$ & $36(1)$ \\
\hline $\mathrm{O} 2$ & $4231(3)$ & $3017(4)$ & $2310(2)$ & $60(1)$ \\
\hline 03 & $2961(3)$ & $4766(3)$ & $478(2)$ & $45(1)$ \\
\hline 04 & $5151(3)$ & $4209(4)$ & $627(2)$ & $53(1)$ \\
\hline
\end{tabular}

ends of the molecule contain $\mathrm{C} 3$ to $\mathrm{C} 12$ and $\mathrm{C} 16$ to $\mathrm{C} 25$, respectively. The angle between them is about $86.2(3)^{\circ}$, revealing a tilted and/or twisted conformation of the molecule (Fig. 2). All of the bond lengths and angles within the central 20-membered ring are listed in Table 3. In the crystal packing, the shortest contact is between $\mathrm{O} 4$ and $\mathrm{C} 14^{\prime}$ ( $3.17 \AA$ ) , which may be caused by an electrostatic interaction between two ester groups (Fig. 3). Further short contacts are C23-C23' (3.55 A) and $\mathrm{C} 22-\mathrm{C} 24^{\prime}(3.57 \AA)$, which rest on $\pi$-electron interactions between the acetylene/phenyl subunits of neighbouring molecules. $\mathrm{C} 14^{\prime}$ and $\mathrm{C} 23^{\prime}, \mathrm{C} 24^{\prime}$ were generated by two different inversion centers, respectively. Together with their
Table 3 Bond lengths $[\AA]$ and angles $\left[{ }^{\circ}\right]$ of the 20-membered ring

\begin{tabular}{llll}
\hline $\mathrm{C} 1-\mathrm{O} 1$ & $1.328(5)$ & $\mathrm{C} 14-\mathrm{O} 3$ & $1.316(5)$ \\
$\mathrm{C} 1-\mathrm{C} 2$ & $1.505(5)$ & $\mathrm{C} 14-\mathrm{C} 15$ & $1.494(6)$ \\
$\mathrm{C} 2-\mathrm{C} 3$ & $1.522(6)$ & $\mathrm{C} 15-\mathrm{C} 16$ & $1.543(6)$ \\
$\mathrm{C} 3-\mathrm{C} 4$ & $1.505(5)$ & $\mathrm{C} 16-\mathrm{C} 17$ & $1.497(6)$ \\
$\mathrm{C} 4-\mathrm{C} 9$ & $1.405(6)$ & $\mathrm{C} 17-\mathrm{C} 22$ & $1.412(5)$ \\
$\mathrm{C} 9-\mathrm{C} 10$ & $1.438(6)$ & $\mathrm{C} 22-\mathrm{C} 23$ & $1.444(5)$ \\
$\mathrm{C} 10-\mathrm{C} 11$ & $1.191(6)$ & $\mathrm{C} 23-\mathrm{C} 24$ & $1.199(5)$ \\
$\mathrm{C} 11-\mathrm{C} 12$ & $1.462(7)$ & $\mathrm{C} 24-\mathrm{C} 25$ & $1.469(5)$ \\
$\mathrm{C} 12-\mathrm{C} 13$ & $1.514(7)$ & $\mathrm{C} 25-\mathrm{C} 26$ & $1.505(6)$ \\
$\mathrm{C} 13-\mathrm{O} 3$ & $1.446(6)$ & $\mathrm{C} 26-\mathrm{O} 1$ & $1.444(5)$ \\
& & & \\
$\mathrm{O} 1-\mathrm{C} 1-\mathrm{C} 2$ & $111.6(3)$ & $\mathrm{C} 14-\mathrm{C} 15-\mathrm{C} 16$ & $115.6(4)$ \\
$\mathrm{C} 1-\mathrm{C} 2-\mathrm{C} 3$ & $114.3(3)$ & $\mathrm{C} 17-\mathrm{C} 16-\mathrm{C} 15$ & $110.6(3)$ \\
$\mathrm{C} 4-\mathrm{C} 3-\mathrm{C} 2$ & $112.3(3)$ & $\mathrm{C} 22-\mathrm{C} 17-\mathrm{C} 16$ & $121.4(3)$ \\
$\mathrm{C} 9-\mathrm{C} 4-\mathrm{C} 3$ & $120.7(4)$ & $\mathrm{C} 17-\mathrm{C} 22-\mathrm{C} 23$ & $118.4(3)$ \\
$\mathrm{C} 4-\mathrm{C} 9-\mathrm{C} 10$ & $120.5(4)$ & $\mathrm{C} 24-\mathrm{C} 23-\mathrm{C} 22$ & $177.3(4)$ \\
$\mathrm{C} 11-\mathrm{C} 10-\mathrm{C} 9$ & $177.1(5)$ & $\mathrm{C} 23-\mathrm{C} 24-\mathrm{C} 25$ & $176.7(4)$ \\
$\mathrm{C} 10-\mathrm{C} 11-\mathrm{C} 12$ & $178.4(6)$ & $\mathrm{C} 24-\mathrm{C} 25-\mathrm{C} 26$ & $112.4(4)$ \\
$\mathrm{C} 11-\mathrm{C} 12-\mathrm{C} 13$ & $112.6(4)$ & $\mathrm{O} 1-\mathrm{C} 26-\mathrm{C} 25$ & $107.2(3)$ \\
$\mathrm{O} 3-\mathrm{C} 13-\mathrm{C} 12$ & $105.3(4)$ & $\mathrm{C} 1-\mathrm{O} 1-\mathrm{C} 26$ & $117.4(3)$ \\
$\mathrm{O} 3-\mathrm{C} 14-\mathrm{C} 15$ & $112.7(4)$ & $\mathrm{C} 14-\mathrm{O} 3-\mathrm{C} 13$ & $116.2(4)$ \\
\hline
\end{tabular}

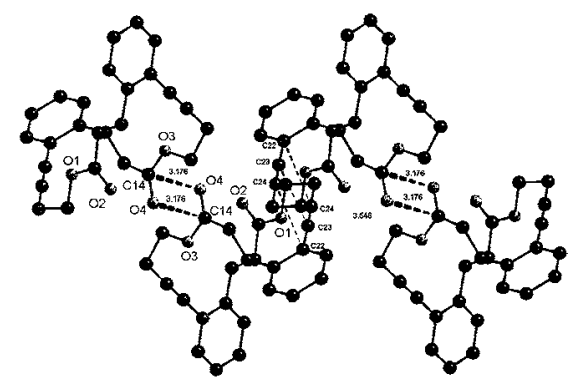

Fig. 3 Packing arrangement of $\mathbf{3}$ in the crystal. The numbers are the inter atomic distances in $\AA$.

equivalents generated by centres of symmetry, these contacts form layers parallel to the (001) lattice plane (Fig. 3). Perpendicular to this plane, however, there are only weak interactions. Due to this fact, the $c$-axis is markedly longer than $a$ and $b$, which is in good agreement with a well-known crystallographic concept. ${ }^{5}$

The crystal selected for our analysis was twinned to (001)[103] with one component (96\%) very dominating. Twinning is a sign of disorder in crystals, and especially here it reveals that the directing forces for crystal growth are rather weak along [001].

\section{References}

1. A. Parenty, X. Moreau, and J.-M. Campagne, Chem. Rev., 2006, 106, 911.

2. P. Wessig, G. Müller, A. Kühn, R. Herre, H. Blumenthal, and S. Troelenberg, Synthesis, 2005, 1445.

3. P. Wessig and G. Müller, Chem. Commun., 2006, 4524.

4. J. Inanaga, K. Hirata, H. Saeki, T. Katsuki, and M. Yamaguchi, Bull. Chem. Soc. Jpn., 1979, 52, 1989.

5. P. Hartmann and W. G. Perdok, Acta Cryst., 1955, 8, 49. 